\title{
Implementation of multi-mode nursing insulation program for patients receiving surgery for spine tumor: a propensity score-matched analysis
}

Juan Liu*, Chunyan Gao, Hailong Fu, Xiaonan Zhou, Li Zhang, Xiaomei Tang, Yanru Wu, Hui Zhu, Sisi Yang, Yafeng Qu, Yajuan Yang and Haiqin Yang

\begin{abstract}
Background: Spinal tumor surgery usually involved long operation time, large area of soft tissue resection and long wound, and was prone to hypothermia during the operation. Therefore, actively promoting insulation and optimizing the intraoperative insulation program have great potential in reducing the incidence of hypothermia and reducing the incidence of postoperative complications. In this study, we compared patients who did not implement multimode nursing insulation program (MNIP) with those who implemented MNIP, observing and comparing clinical outcomes, and complications in both groups, with the aim of developing an optimal management plan for the preoperative, intraoperative, and postoperative periods, respectively.
\end{abstract}

Methods: We selected 2 periods of 1 year, before $(n=120$ patients) and after MINP implementation $(n=120$ patients). Data were collected on patient demographics, operative, perioperative details, temperature changes, anesthesia recovery effect, incidence of postoperative wound infection, length of hospital stay and complications. PS analyses were used for dealing with confounding bias in this retrospective observational study.

Results: After PS matching, the outcomes of 120 well-balanced pairs of patients were compared (No-MNIP vs MNIP). There was no significant difference concerning the satisfaction survey. The results indicated that the MNIP had better insulation effect at $90 \mathrm{~min}, 120 \mathrm{~min}, 150 \mathrm{~min}$ after anesthesia induction and after surgery. There were 16 cases of complications in the No-MNIP group and 5 cases in the MNIP group postoperative, which have significant statistical difference.

Conclusion: In this study, the incidence of intraoperative hypothermia was effectively reduced by adopting the multi-mode insulation scheme, thus reducing the incidence of incision infection and shortening the length of hospital stay of patients.

Keywords: Spine tumor, Enhanced recovery after surgery, Hypothermia, Multi-mode nursing insulation program, Propensity score-matched

\section{Background}

With the continuous improvement of the average life expectancy of the population, the incidence of spinal tumors is increasing year by year. Spinal tumors are mainly divided into primary tumors and metastatic 
tumors. Primary spinal tumors are rare, accounting for about 4.6 to $8.8 \%$ of all bone tumors, and spinal metastases account for $50 \%$ of all bone metastases. It has been reported in the literature that 10 to $30 \%$ of patients with primary malignant tumors will have spinal metastasis at an advanced stage. Intractable pain, spinal instability and nerve compression are the most common symptoms of spinal tumor. $30 \%$ of patients with spinal tumor will seriously affect the quality of life, have paraplegia and even shorten the survival period. The main goal of surgical treatment is to remove the tumor, relieve nerve compression and rebuild the stability of the spine. Spinal tumor surgery usually involved long operation time, large area of soft tissue resection and long wound, and was prone to hypothermia during the operation [1-6].

Perioperative hypothermia means that the core body temperature of surgical patients was lower than $36{ }^{\circ} \mathrm{C}$, and the incidence was about $50-90 \%$. It could increase the infection rate of surgical incisions, the incidence of postoperative complications, and prolong the patient's hospital stay. In United States, postoperative infection had become the 4th leading killer, and its death toll exceeded the combined number of AIDS, breast cancer and traffic accidents. Its annual medical expenses were 1-10 billion dollars. CMS-SCIP proposed the best strategy to prevent surgical site infections: proper hair removal, reasonable preventive use of antibiotics, maintenance of normal body temperature, and blood glucose control. It could be seen that maintaining a normal body temperature during the perioperative period had an important significance in preventing infections at the surgical site. Huang conducted a survey of 28 research centers and found that passive thermal insulation measures were less than $90 \%$ and active thermal insulation measures were less than $10 \%$ in various stages of thermal insulation methods. The incidence of hypothermia during the perioperative period of general anesthesia patients was as high as $44.5 \%$. Therefore, actively promoting active insulation and optimizing the intraoperative insulation program have great potential in reducing the incidence of hypothermia and reducing the incidence of postoperative complications [7-14].

Enhanced Recovery After Surgery (ERAS) is the core ideology leading the development of field of surgrical treatment [15-19]. However, there is still no idea about the application of the ERAS concept in nursing insulation program. Our hospital began the implementation of a multi-mode nursing insulation program (MNIP) regarding the development of ERAS for patients receiving surgery for spine tumors in September 2019. The MNIP aimed to minimize and avoided the occurrence of hypothermia during spinal tumor surgery, and minimized infection and orther complications.
In this study, we used propensity score-matched (PS) analysis to compare the controlled group patients MNIP with those patients received MNIP, observing and comparing clinical outcomes, and complications, and also aimed to develope an optimal treatment plan.

\section{Patients and methods}

Inclusion and exclusion criteria

The study was approved by the Institutional Ethics Committee of Changzheng Hospital. The inclusion criterias were as follows: (1) patients were diagnosed as with spinal primary malignant tumors or metastatic tumors; (2) survival is expected to be longer than six months. The exclusion criterias were as follows: (1) patients with malignant metastatic tumors who cannot tolerate surgery or undergo palliative surgery; (2) having metastases in the lungs or other organs; (3) survival is expected to be less than six months. All the surgeries were performed by the same group of surgeons.

The MNIP multi-disciplinary team comprised of spine surgeons, anesthesiologists, physiotherapists, and nurses finalized the MNIP. This led us to selected 2 periods of 1 year, before (Group No-MNIP, from May 2019 to Apr 2020, $n=120$ patients) and after implementing of the ERAS (Group MNIP, from May 2020 to Apr 2020, $\mathrm{n}=120$ patients).

\section{Multi-mode nursing insulation program}

Before the patient arrived in the operating room, the room temperature was preheated at $22-24{ }^{\circ} \mathrm{C}$, and the specially-made segmented nine-holes with a length of $1.2 \mathrm{~m}$ and width of $1.0 \mathrm{~m}$ were preheated and covered to keep warm; the pre-heated segmented thermal insulation surgical gown was used during the operation. Used air heaters to heat and inflatable heat preservation, adjust at any time according to the patient's body temperature, to ensured that the patient's body temperature was above $36{ }^{\circ} \mathrm{C}$; used an infusion warming instrument for infusion; recorded the disinfection time, the amount of input and output, the temperature of the rinsing solution, and implement targeted preventive measures for patients who were prone to hypothermia. Active warmth and passive warmth were synchronized; body thermometers were continuously monitored.

\section{Evaluation outcome}

Patient demographics, clinical history, comorbidities, and operative details were noted from the hospital records. The outcome measures for the study were as following:

\section{Temperature changes during surgery}

The rectal temperature was recorded after induction of anesthesia (T1), $30 \mathrm{~min}$ (T2), $60 \mathrm{~min}$ (T3), $90 \mathrm{~min}$ (T4), 
120 min (T5), 150 min (T6), and 1 day after operation (T7).

\section{Evaluation of anesthesia recovery effect}

The Steward recovery score was used to evaluate the anesthesia recovery effect of the two groups of patients after the operation, and the time for the patients to recover from anesthesia after the operation was recorded. Only patients with a score of 4 or more could be allowed to leave the operating room or recovery room. Patients with intraoperative hypothermia could cause prolonged recovery time of anesthesia, and the recovery time of patients after surgery was generally within $60-90 \mathrm{~min}$. If the recovery time exceeded this time limit, it would be regarded as delayed recovery.

\section{Incidence of postoperative wound infection and length of hospital stay}

Closely observed the wound healing of patients after surgery, and recorded the number of delayed wound healing and infections, as well as the number of days the patient was hospitalized.

\section{Complications}

Closely observed the patient's reaction, whether there were lung infections, urinary tract infections, bedsores, deep vein thrombosis, pulmonary embolism, cerebral vascular accident, gastrointestinal and myocardial infarction, establish an adverse reaction observation table, record the adverse events and their manifestations, start time, stop time and remission reasons.

\section{Satisfactions}

The satisfactions of patients were surveyed by telephone or email according to a Likert scale.

\section{Statistical analysis}

SPSS 22.0 statistical software (SPSS Inc., Chicago, Illinois) was used. Efficacy and safety analyses will be conducted according to the intention-to-treat principle using the "last observation carried forward" rule. A $P$ value of less than 0.05 is defined as statistically significant with 2-sided 90\% CIs.

\section{Results}

\section{Results of PS matching}

After PS matching, outcomes were compared for 80 well-balanced pairs of patients, respectively (No-MNIP group and MNIP group). The baseline demographic details, clinical characteristics, and relevant operative information of the 160 patients in the study groups were depicted. There were no baseline statistical differences between the No-MNIP group and the MNIP group in demographic characteristics (Table 1).

\section{Follow-up}

This study included a 3-month follow-up period. The rectal temperature was recorded after induction of anesthesia (T1), $30 \mathrm{~min}$ (T2), $60 \mathrm{~min}$ (T3), $90 \mathrm{~min}$ (T4), $120 \mathrm{~min}$ (T5), $150 \mathrm{~min}$ (T6), and 1 day after operation (T7). Follow-up was completed in all 160 patients, and there were no missed cases.

\section{General results}

There was no significant difference in the baseline VAS scores and Frankel grades in the two study groups. All patients achieved remarkable pain relief (postoperative VAS score range 0-2) and improvement in neurological function (postoperative Frankel grade D or E).

\section{Evaluation of temperature changes during surgery}

A comparison between the No-MNIP group and MNIP group concerning various temperatures during surgery was depicted in Table 2. Patients in the MNIP group had a significantly higer temperature as compared to the NoMNIP group at T4-7 point. $(\mathrm{p}<0.05)$ The above results indicated that the MNIP had better insulation effect at $90 \mathrm{~min}, 120 \mathrm{~min}, 150 \mathrm{~min}$ after anesthesia induction and after surgery. (Table 2).

\section{Evaluation of anesthesia recovery effect}

The results showed that there was significant difference between the two groups in the recovery time of postoperative anesthesia recovery, with statistical significance $(\mathrm{P}<0.05)$. The scores of anesthesia recovery ability in the MNIP group were significantly improved, indicating that the cardiopulmonary function and circulation metabolism of patients were improved after the multi-mode insulation intervention, and the anesthesia time was significantly shortened (Table 3 ).

\section{Evaluation of incidence of postoperative wound infection and length of hospital stay}

It was observed that only 5 cases of postoperative wound infection/ delayed wound healing occurred in the MNIP group, which was lower than 12 cases in the No-MNIP group. In addition, the wound infection/ delayed wound healing rate of patients in the MNIP group group was also shorter $(\mathrm{P}<0.05)$, thus the result indicated that MNIP reduced the length of postoperative hospital stay. (Table 4).

\section{Complications survey}

The summary of postoperative complications in 30 days was depicted in Table 5. There were 16 cases 
Table 1 Patient characteristics of propensity score-matched patient groups

\begin{tabular}{|c|c|c|c|}
\hline \multirow[t]{2}{*}{ Variables } & \multicolumn{2}{|l|}{ All patients $(\mathrm{N}=240)$} & \multirow[t]{2}{*}{$d$} \\
\hline & No-MNIP Group $(n=120)$ & MNIP Group $(n=120)$ & \\
\hline Age & $62.3 \pm 8.3$ & $63.1 \pm 8.2$ & 0.23 \\
\hline Gender (M) & 64 & 62 & 0.14 \\
\hline Tumor state (primary/metastasis) & $102 / 18$ & $99 / 22$ & 0.23 \\
\hline Duration of symptom (m) & $3.3 \pm 1.3$ & $3.1 \pm 1.2$ & 0.54 \\
\hline Chemotherapy (cases) & 62 & 60 & 0.21 \\
\hline Radiotherapy (cases) & 24 & 20 & 0.19 \\
\hline Pre-SINS score & $8.3 \pm 3.1$ & $8.6 \pm 2.9$ & 0.33 \\
\hline Operating time (h) & $4.6 \pm 2.1$ & $4.8 \pm 1.1$ & 0.21 \\
\hline Blood loss (ml) & $1230.5 \pm 180.2$ & $1250.2 \pm 190.5$ & 0.31 \\
\hline \multirow[t]{2}{*}{ Variables } & \multicolumn{2}{|l|}{ Marched patients $(\mathrm{N}=160)$} & \multirow[t]{2}{*}{$d$} \\
\hline & No-MNIP Group $(n=80)$ & MNIP Group $(n=80)$ & \\
\hline Age & $61.0 \pm 5.2$ & $60.2 \pm 4.9$ & \\
\hline Gender (M) & 39 & 42 & \\
\hline Tumor state (primary/metastasis) & $70 / 10$ & $68 / 12$ & 0.02 \\
\hline Duration of symptom (m) & $3.6 \pm 1.1$ & $3.2 \pm 1.5$ & 0.01 \\
\hline Chemotherapy (cases) & 40 & 38 & 0.03 \\
\hline Radiotherapy(cases) & 10 & 12 & 0.16 \\
\hline Pre-SINS score & $8.5 \pm 2.8$ & $8.4 \pm 3.1$ & 0.01 \\
\hline Operating time (min) & $4.5 \pm 2.5$ & $4.7 \pm 2.5$ & 0.08 \\
\hline Blood loss (ml) & $1300.5 \pm 200.2$ & $1200.5 \pm 180.2$ & 0.03 \\
\hline
\end{tabular}

Bold indicate when the results of the comparisons between the two groups were statistically significant; $d$, standardized difference

Table 2 Evaluation of temperature changes during surgery

\begin{tabular}{lllc}
\hline Time Point & $\begin{array}{l}\text { No-MNIP } \\
\text { Group } \\
(\mathbf{n}=\mathbf{1 2 0})\end{array}$ & MNIP Group $(\mathbf{n}=\mathbf{1 2 0})$ & $\boldsymbol{P}$ \\
\hline T1 & $36.89 \pm 0.34$ & $36.90 \pm 0.30$ & 0.632 \\
T2 & $36.62 \pm 0.45$ & $36.91 \pm 0.34$ & 0.313 \\
T3 & $36.23 \pm 0.36$ & $36.67 \pm 0.36$ & 0.329 \\
T4 & $35.88 \pm 0.27$ & $36.50 \pm 0.42$ & $\mathbf{0 . 0 1 2 *}$ \\
T5 & $35.72 \pm 0.28$ & $36.44 \pm 0.47$ & $\mathbf{0 . 0 2 4}$ \\
T6 & $35.61 \pm 0.34$ & $36.44 \pm 0.47$ & $<\mathbf{0 . 0 0 1 *}$ \\
T7 & $35.24 \pm 0.89$ & $36.44 \pm 0.66$ & $<\mathbf{0 . 0 0 1 *}$ \\
\hline
\end{tabular}

The bold values indicated when the results of the comparisons between the two groups were statistically significant

*Indicate when the results of the comparisons between the two groups were statistically significant

of complications in the No-MNIP group and 5 cases in the MNIP group postoperative, which have significant statistical difference. 5 cases and 2 cases occurred deep venous thrombosis in two groups, respectively. 6 cases and 2 cases occurred urinary tract infection in two groups, respectively. 3 cases and 1 cases occurred respiratory tract infection in two groups, respectively. 1 case occurred gastrointestinal in the No-MNIP group. 1 case
Table 3 Evaluation of anesthesia recovery effect

\begin{tabular}{lllr}
\hline Variables & $\begin{array}{l}\text { No-MNIP } \\
\text { Group } \\
(\mathbf{n}=\mathbf{1 2 0})\end{array}$ & $\begin{array}{l}\text { MNIP Group } \\
(\mathbf{n}=\mathbf{1 2 0})\end{array}$ & $P$ \\
\hline Steward score & $2.75 \pm 2.11$ & $3.81 \pm 1.54$ & $\mathbf{0 . 0 2 3 ^ { * }}$ \\
Recovery time (h) & $1.12 \pm 0.55$ & $0.89 \pm 0.37$ & $\mathbf{0 . 0 1 3 ^ { * }}$ \\
Recovery delay rate & $15.65 \%$ & $6.76 \%$ & $<\mathbf{0 . 0 0 1 *}$ \\
(\%) & & \\
\hline $\begin{array}{l}\text { The bold values indicated when the results of the comparisons between the two } \\
\text { groups were statistically significant }\end{array}$ \\
*Indicate when the results of the comparisons between the two groups were \\
statistically significant
\end{tabular}

occurred myocardial infarction in the No-MNIP group. No deaths, no readmission, and no reoperation occurred in either group. (Table 5).

\section{Satisfaction survey}

Summary of the satisfaction survey was depicted in Table 5. There was no significant difference in "Overall surgical result", "Current health status", "Quality of cares", "Satisfaction about LOS", "I would redo it" and "I would advise it to a relative" scores in the two study groups, respectively. ( $p>0.05$ ) (Table 6). 
Table 4 Evaluation of incidence of postoperative wound infection and length of hospital stay

\begin{tabular}{llcl}
\hline Variables & $\begin{array}{l}\text { No-MNIP } \\
\text { Group } \\
(\mathbf{n}=\mathbf{1 2 0})\end{array}$ & MNIP Group $(\mathbf{n = 1 2 0 )})$ & $P$ \\
\hline $\begin{array}{l}\text { Incidence of delayed } \\
\text { wound healing and } \\
\text { infections ( } \mathrm{n}, \%)\end{array}$ & $\mathbf{0 . 0 3 1}$ * \\
$\begin{array}{l}\text { Length of hospital } \\
\text { stay (d) }\end{array}$ & $5.67 \pm 4.15$ & $4.35 \pm 3.67$ & $\mathbf{0 . 0 1 3}$ \\
\hline $\begin{array}{l}\text { The bold values indicated when the results of the comparisons between the two } \\
\text { groups were statistically significant } \\
\text { *Indicate when the results of the comparisons between the two groups were } \\
\text { statistically significant }\end{array}$
\end{tabular}

\section{Discussion}

There was no single intervention to prevent hypothermia that may occur during surgery. During the operation, it was often necessary to adopt a multi-mode comprehensive thermal insulation scheme, which was actively adjusted according to the dynamic changes of the patient's body temperature during the operation, so as to effectively reduce the incidence of postoperative complications and ensure the perioperative safety of patients. In order to exclude the influence of ambient temperature on the change of body temperature of patients, the operating room temperature in the control group and the comprehensive insulation group was set at $22-24{ }^{\circ} \mathrm{C}$ in this study. Intravenous heating liquid could make the patient's body temperature rise, and the effect would be better combined with other insulation measures. Heating devices for active insulation included infrared lamps, electric blankets, blankets or warm water circulating beds, hot air blowers or convective air heating systems. Air heating was the most studied intervention measure at present. It could increase the central temperature by $0.5-1{ }^{\circ} \mathrm{C}$, which had good heating effect. It could also reduce heart complications, reduce the probability of infection and wound healing, and reduce the incidence of postoperative shivering. In this study, the incidence of
Table 5 Comparison of complications between the two groups

\begin{tabular}{lll}
\hline Variables & $\begin{array}{l}\text { No-MNIP Group } \\
(\mathbf{n}=\mathbf{1 2 0})\end{array}$ & $\begin{array}{l}\text { MNIP } \\
\text { Group } \\
(\mathbf{n}=\mathbf{1 2 0})\end{array}$ \\
\hline Infection & 0 & 0 \\
Deep venous thrombosis & 5 & 2 \\
Urinary tract infection & 6 & 2 \\
Respiratory tract infection & 3 & 1 \\
Pulmonary embolism & 0 & 0 \\
Cerebral vascular accident & 0 & 0 \\
Gastrointestinal & 1 & 0 \\
Myocardial infarction & 1 & 0 \\
30-Day readmission & 0 & 0 \\
30- to 90-Day readmission & 0 & 0 \\
Reoperation & 0 & 0 \\
\hline
\end{tabular}

hypothermia in the MNIP group was significantly lower than that in the conventional insulation group, suggesting that the insulation measures adopted in this study had good effects and could reduce the incidence of hypothermia in patients [14, 20-23].

Perioperative hypothermia could lead to increase intraoperative blood loss, prolong postoperative anesthesia recovery time, increased heart rate, increased oxygen consumption, impaired immune function, and increas postoperative complications (such as coagulation dysfunction, arrhythmia, wound infection). During general anesthesia, the threshold of hypothalamic thermoregulatory center response to hypothermia was lowered by general anesthesia. In addition, anesthetic drugs had an inhibitory effect on the central self-regulation system that maintained normal body temperature. Muscle relaxants woud block thermogenesis and hypothermia, lead to thermoregulation of vasoconstriction, reduce subcutaneous oxygen tension and cause tissue hypoxia, and indirectly inhibit the function of neutrophils, thus inhibiting the immune function of the body. Hypothermia aggravates the consumption of postoperative protein and affects wound healing,

Table 6 Comparison of satisfaction between the two groups

\begin{tabular}{ll}
\hline Variables & No-MNIP Group (n=120) \\
\hline Overall surgical result (satisfied or very satisfied), (\%) & $\begin{array}{l}\text { MNIP } \\
\text { Group } \\
\text { (n= 120) }\end{array}$ \\
Current health status (satisfied or very satisfied), (\%) & 58 \\
Quality of cares (satisfied or very satisfied), (\%) & 60 \\
Satisfaction about LOS (agree or strongly agree), (\%) & 50 \\
I would redo it (agree or strongly agree), (\%) & 53 \\
I would advise it to a relative (agree or strongly agree), (\%) & 50 \\
\hline
\end{tabular}


decreased platelet function, impaired coagulation function, increased blood viscosity, and even DIC, caused electrolyte disruptions that can lead to serious complications such as arrhythmias. Based on some studies, the World Health Organization issued surgical safety guidelines and clearly stated that, maintaining normal intraoperative body temperature could effectively reduce the incidence of surgical site infection (SSI) after surgery. Since then, various complications caused by intraoperative hypothermia and effective means of thermal insulation have attracted more and more attention and research in the medical field. In this study, the incidence of intraoperative hypothermia was effectively reduced by adopting the multi-mode insulation scheme, thus reducing the incidence of incision infection and shortening the length of hospital stay of patients [24-28].

At present, there were many methods of intraoperative insulation, but many effective insulation measures had not been effectively promoted due to the high price of the instrument and cumbersome operation. Natural insulation was still the main prevention measure of hypothermia in the majority of primary hospitals, and the effect of such methods was limited. In addition, with the rapid development of medical technology, more and more complex operations have been carried out in clinical practice, and the factors causing intraoperative hypothermia have become more and more complicated. How to take more effective preventive measures to reduce the incidence of intraoperative hypothermia needed further study and discussion. Taking this as the starting point, this study developed and optimized the all-round multimode operating room thermal insulation plan, and created a multi-mode thermal insulation plan from various processes such as environment, personnel, facilities and equipment, and operating norms, so as to provide the optimal thermal insulation for patients during operation.

\section{Conclusion}

In this study, the incidence of intraoperative hypothermia was effectively reduced by adopting the multi-mode insulation scheme, thus reducing the incidence of incision infection and shortening the length of hospital stay of patients.

\footnotetext{
Abbreviations

ERAS: Enhanced recovery after surgery; MNIP: Multi-mode nursing insulation program; PS analysis: Propensity score-matched analysis.
}

Acknowledgements

Not applicable.

\section{Authors' contributions}

LJ, GCY are co-first authors of this manuscript, contributing equally to the design, conduct of the trials and drafting the manuscript. ZXN, ZL, TXM and WYR are responsible for recruiting the participants. FHL, ZH, YSS and QYF participated in the statistical design. $\mathrm{YYJ}$ and $\mathrm{YHQ}$ are co-responding authors of this manuscript. All authors read and approved the final manuscript.

\section{Funding}

This work was sponsored by research grants from the Projects Of Changzheng Hospital (CZYY-HLQ98; CZYY-HLZ99); the Pyramid Talent Training Projects From Changzheng Hospital (2018).

\section{Availability of data and materials}

The datasets used and/or analyzed during the current study are available from the corresponding author on reasonable request. Ethics approval and consent to participate. The study design, procedures, and informed consent procedure were approved by the Changzheng Hospital. Consent to participate will be obtained from the participants.

\section{Declarations}

\section{Ethics approval and consent to participate}

This study is to be conducted following the principles of the Declaration of Helsinki and has been approved by Ethics Review Committee.

\section{Consent for publication}

Not applicable.

\section{Competing interests}

The authors declare that they have no competing interests.

Received: 27 October 2021 Accepted: 30 December 2021

Published online: 08 January 2022

\section{References}

1. Honda A, lizuka Y, Okamoto M, et al. Malignant peripheral nerve sheath tumor of the cervical spine treated with surgical resection followed by $X$-ray radiotherapy or carbon ion radiotherapy: a report of three cases. Spine Surg Relat Res. 2020;4(3):269-73.

2. Dupuis M, Shen Y, Curcio C, et al. Successful treatment of lipofibromatosis-like neural tumor of the lumbar spine with an NTRK-fusion inhibitor. Clin Sarcoma Res. 2020;10:14.

3. Kim YJ, Hong JH, Park JH, Cho SJ. Tenosynovial giant cell tumor of the upper cervical spine arising from the posterior atlanto-occipital membrane: a case report. Skeletal Radiol. 2021;50(2):451-5.

4. Smith JL, Christopher AN, Evans NR 3rd. Novel two-stage open spine and robotic thoracic approach to resection of a mediastinal tumor. Ann Thorac Surg. 2021;111(1):e27-9.

5. Singh J, Bahadur R, Garg S, Rajpal K, Chopra K. Clinical outcome in giant cell tumor of cervico-thoracic spine: our experience with three cases. Int J Surg Case Rep. 2020;72:45-51.

6. Baig Mirza A, Visagan R, Reisz Z, Bodi I, Bell D, Grahovac G. Spinal cord compression caused by fibroblastic reticular cell tumor (FRCT) originating from thoracic spine. World Neurosurg. 2020;141:20-4.

7. Oner Cengiz H, Ucar S, Yilmaz M. The role of perioperative hypothermia in the development of surgical site infection: a systematic review. AORN J. 2021;113(3):265-75.

8. Sari S, Aksoy SM, But A. The incidence of inadvertent perioperative hypothermia in patients undergoing general anesthesia and an examination of risk factors. Int J Clin Pract. 2021;75(6): e14103.

9. Dagli R, Celik F, Ozden H, Sahin S. Does the laminar airflow system affect the development of perioperative hypothermia? A randomized clinical trial. HERD. 2021;14(3):202-14.

10. Mendonca FT, Ferreira JDS, Guilardi VHF, Guimaraes GMN. Prevalence of inadvertent perioperative hypothermia and associated factors: a crosssectional study. Ther Hypothermia Temp Manag. 2021. https://doi.org/10. 1089/ther.2020.0038. 
11. Urfalioglu A, Urfalioglu S, Oksuz G, et al. The effects of active warming on perioperative inadvertent hypothermia in patients undergoing vitreoretinal surgery under local anesthesia. Indian J Ophthalmol. 2021;69(2):308-13.

12. Tanner J, Timmons S, Bayston R, Adams K, Baxendale B. Using a comprehensive audit to identify local context prior to care bundle design and implementation for inadvertent perioperative hypothermia in colorectal surgery. BMJ Open Qual. 2021. https://doi.org/10.1136/ bmjoq-2020-001132.

13. Wagner D, Hooper V, Bankieris K, Johnson A. The relationship of postoperative delirium and unplanned perioperative hypothermia in surgical patients. J Perianesth Nurs. 2021;36(1):41-6.

14. Lee JH, Heo HJ, Kim YY, Baek SM, Kim KM, Jung DW. The effect of interscalene brachial plexus block with propofol sedation on preventing perioperative hypothermia during arthroscopic shoulder surgery. Korean J Anesthesiol. 2021;74(1):53-8.

15. Cristobal Poch L, Cagigas Fernandez C, Gomez-Ruiz M, et al. Implementation of an enhanced recovery after surgery program with robotic surgery in high-risk patients obtains optimal results after colorectal resections. J Robot Surg. 2021. https://doi.org/10.1007/s11701-021-01281-w.

16. Li R, Wang K, Qu C, et al. The effect of the enhanced recovery after surgery program on lung cancer surgery: a systematic review and metaanalysis. J Thorac Dis. 2021;13(6):3566-86.

17. Sanchez-Iglesias JL, Gomez-Hidalgo NR, Perez-Benavente A, et al. ASO visual abstract: importance of enhanced recovery after surgery protocol compliance on length of stay in ovarian cancer surgery. Ann Surg Oncol. 2021. https://doi.org/10.1245/s10434-021-10258-w.

18. Duojun W, Hui Z, Zaijun L, Yuxiang G, Haihong C. Enhanced recovery after surgery pathway reduces the length of hospital stay without additional complications in lumbar disc herniation treated by percutaneous endoscopic transforaminal discectomy. J Orthop Surg Res. 2021;16(1):461.

19. Lunel T, Mohkam K, Merle P, Impact of, et al. Enhanced recovery after surgery (ERAS) recommendations on outcomes after hepatectomy in cirrhotic and non-cirrhotic patients. World J Surg. 2016. https://doi.org/ 10.1016/j.hpb.2021.08.255.

20. Honkavuo L, Loe SAK. Nurse anesthetists' and operating theater nurses' experiences with inadvertent hypothermia in clinical perioperative nursing care. J Perianesth Nurs. 2020;35(6):676-81.

21. Esen O, Yilmaz G, Aydin N. Perioperative hypothermia in pediatric patients operated in a tertiary care center: incidence and correlates. Pak J Med Sci. 2020;36(4):793-8.

22. Sorber $\mathrm{R}$, Crawford TC, Wolfgang CL, Rizkalla N, Frank SM, Atallah C. Hypothermia prevention in hepatopancreatobiliary surgery through a multidisciplinary perioperative protocol: a case-control, propensitymatched study. J Clin Anesth. 2020;65: 109858.

23. Nordgren M, Hernborg $O$, Hamberg A, Sandstrom E, Larsson G, Soderstrom $L$. The effectiveness of four intervention methods for preventing inadvertent perioperative hypothermia during total knee or total hip arthroplasty. AORN J. 2020;111(3):303-12.

24. Hanna M, Htun Z, Islam S, Hanna N, Kothari U, Nayak A. A quality improvement initiative to improve perioperative hypothermia rates in the NICU utilizing checklists. Pediatr Qual Saf. 2020;5(5): e367.

25. Wang R, Ping FM, Liu YM, et al. Effect of competence health cultivation on the prevention and control of inadvertent perioperative hypothermia. Rev Assoc Med Bras (1992). 2020;66(7):924-30.

26. Koleini E, Cohen JS, Darwish OM, Pourafkari L, Rein L, Nader ND. Perioperative hypothermia after transurethral surgeries: is it necessary to heat the irrigation fluids? Turk J Anaesthesiol Reanim. 2020;48(5):391-8.

27. Senkal S, Kara U. Guideline implementation and raising awareness for unintended perioperative hypothermia: single-group "before and after" study. Ulus Travma Acil Cerrahi Derg. 2020;26(5):719-27.

28. Rodriguez-Diaz JM, Hayes GM, Boesch J, et al. Decreased incidence of perioperative inadvertent hypothermia and faster anesthesia recovery with increased environmental temperature: a nonrandomized controlled study. Vet Surg. 2020;49(2):256-64.

\section{Publisher's Note}

Springer Nature remains neutral with regard to jurisdictional claims in published maps and institutional affiliations.

Ready to submit your research? Choose BMC and benefit from:

- fast, convenient online submission

- thorough peer review by experienced researchers in your field

- rapid publication on acceptance

- support for research data, including large and complex data types

- gold Open Access which fosters wider collaboration and increased citations

- maximum visibility for your research: over $100 \mathrm{M}$ website views per year

At BMC, research is always in progress.

Learn more biomedcentral.com/submissions 